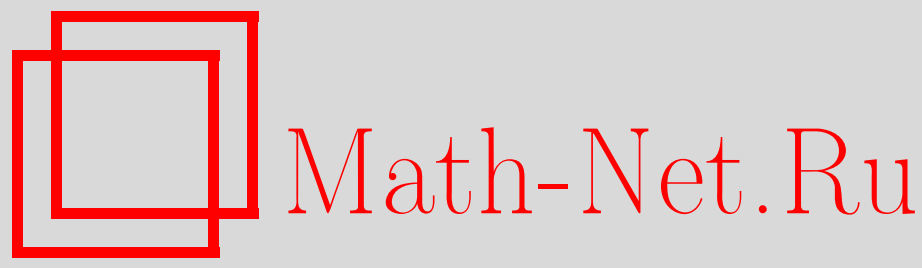

Д. А. Славнов, О квантовой телепортации, ТМФ, 2008, том 157, номер 1, 79-98

DOI: https://doi.org/10.4213/tmf6265

Использование Общероссийского математического портала Math-Net.Ru подразумевает, что вы прочитали и согласны с пользовательским соглашением http://www . mathnet.ru/rus/agreement

Параметры загрузки:

IP : 34.229 .108 .108

26 апреля 2023 г., 17:00:48

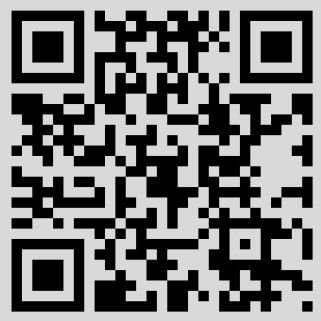




\section{О КВАНТОВОЙ ТЕЛЕПОРТАЦИИ}

В рамках алгебраического подхода рассматривается процедура квантовой телепортации. Выясняется, что для описания этой процедуры не нужно прибегать к гипотезе о нелокальности квантовых измерений. Исследован вопрос о том, какие материальные объекты являются переносчиками информации при квантовой телепортации.

Ключевые слова: запутанные состояния, телепортация квантового состояния, телепортация запутанности.

\section{1. ВВЕДЕНИЕ}

Термин “телепортация", вынесенный в название предлагаемой статьи, может вызвать вполне обоснованную настороженность у читателя научного журнала. Уж очень прочно связался данный термин с фантастической и псевдонаучной литературой. Вместе с тем этот термин укоренился в той области науки, которую часто называют "физика квантовой информации" (см., например, сборник [1]). В последние годы эта область интенсивно развивается, и с ней связывают большие надежды на очень интересные практические приложения.

Термином "телепортация" называют специфический способ передачи информации. Даже в сугубо научной литературе этот способ имеет некоторые черты загадочности, так как четко не указывается материальный переносчик информации. Вместо этого делаются ссылки на нелокальность, которая якобы присуща квантовым измерениям.

В настоящей статье делается попытка дать приземленное, наглядное описание телепортации с указанием на то, какой материальный носитель переносит ту или иную информацию. Наше рассмотрение ведется в рамках специального варианта алгебраического подхода к квантовой теории. Подробное описание данного подхода можно найти в статье [2], более сжатое - в статье [3]. В этих статьях использовался индуктивный способ построения теории. Вначале рассматривались физические явления, подмечались присущие им физические закономерности, затем эти закономерности закреплялись в виде математических аксиом. Здесь мы будем предполагать, что

* Московский государственный университет им. М. В. Ломоносова, Москва, Россия. E-mail: slavnov@goa.bog.msu.ru 
читатель уже знаком с феноменологическим обоснованием аксиом, и ограничимся их формулировкой. Кроме того, мы не будем описывать, как из этих аксиом можно получить стандартный математический аппарат квантовой механики. Интересующихся опять-таки отсылаем к статьям [2], [3].

\section{2. ОСНОВНЫЕ ПОЛОЖЕНИЯ ПОДХОДА}

Центральным понятием используемого подхода является "наблюдаемая". Наблюдаемая - это такой атрибут физической системы, для которого с помощью определенной измерительной процедуры можно получить некоторое численное значение. Все наблюдаемые предполагаются безразмерными. Все измерения подразделяются на воспроизводимые и невоспроизводимые, а также на совместимые и несовместимые. Совместимые измерения осуществляются совместимыми измерительными приборами. Если для некоторой группы наблюдаемых существуют совместимые измерительные приборы, то такие наблюдаемые называются совместимыми (одновременно измеримыми).

ПостулАт 1. Наблюдаемые $\hat{A}$ физической системы являются эрмитовыми элементами некоторой $C^{*}$-алгебры [4] $\mathfrak{A},\left(\hat{A} \in \mathfrak{A}, \hat{A}^{*}=\hat{A}\right)$.

Множество наблюдаемых обозначим $\mathfrak{A}_{+}\left(\mathfrak{A}_{+} \subset \mathfrak{A}\right)$.

ПостулАт 2. Множество совместимых наблюдаемых является максимальной действительной ассоциативной коммутативной подалгеброй $\mathfrak{Q}_{\xi}$ алгебры $\mathfrak{A}$ $\left(\mathfrak{Q}_{\xi} \subset \mathfrak{A}_{+}\right)$.

Индекс $\xi$, который пробегает множество $\Xi$, отличает одну такую подалгебру от другой. Для классической системы такая подалгебра одна, для квантовой их бесконечно много [2].

Будем рассматривать множество $\mathfrak{A}_{+}$в качестве математического образа исследуемой физической системы, а множества $\mathfrak{Q}_{\xi}$ в качестве математических образов соответствующих классических подсистем физической системы. Эти классические подсистемы являются открытыми (взаимодействующими между собой) и не имеют собственной динамики. Состояние классической системы - это такой ее атрибут, который однозначно предопределяет результат всех наблюдаемых системы. Поэтому примем следующий постулат.

ПостулАт 3. Состояние классической подсистемы, наблюдаемые которой являются элементами подалгебры $\mathfrak{Q}_{\xi}$, описывается характером этой подалгебры.

Напомним, что характером $\varphi_{\xi}(\cdot)$ ассоциативной коммутативной алгебры называется гомоморфное отображение этой алгебры в множество действительных чисел: $\hat{A} \stackrel{\varphi_{\xi}}{\longrightarrow} \varphi_{\xi}(\hat{A}), \hat{A} \in \mathfrak{Q}_{\xi}$ (см., например, [4]).

Так как наблюдаемые, принадлежащие подалгебре $\mathfrak{Q}_{\xi}$, совместимы между собой, существует множество измерительных приборов, предназначенных для совместимого измерения этих наблюдаемых. Будем говорить, что эти приборы относятся к $\xi$-типу. 
Множество $\mathfrak{A}_{+}$наблюдаемых квантовой системы можно рассматривать как совокупность подмножеств $\mathfrak{Q}_{\xi}$. Поэтому квантовую систему можно рассматривать как совокупность соответствующих открытых классических подсистем. Каждая наблюдаемая квантовой системы принадлежит некоторому подмножеству $\mathfrak{Q}_{\xi}$. Coответственно, если бы нам были известны состояния всех классических подсистем, то мы могли бы предсказать результат измерения любой наблюдаемой квантовой системы. Исходя из этого назовем элементарным состоянием физической системы совокупность $\varphi=\left[\varphi_{\xi}\right](\xi \in \Xi)$ функционалов $\varphi_{\xi}(\cdot)$, каждый из которых является некоторым характером соответствующей подалгебры $\mathfrak{Q}_{\xi}$. Следующий постулат является центральным в описываемом подходе.

ПостулАт 4. Результат каждого индивидуального измерения наблюдаемых физической системы определяется элементарным состоянием этой системы.

В данном постулате не сказано, что результат определяется однозначно. Дело в том, что одна и та же наблюдаемая $\hat{A}$ может одновременно принадлежать нескольким подалгебрам $\mathfrak{Q}_{\xi}$. Поэтому для ее измерения можно использовать приборы разных типов. Мы привыкли к тому, что если прибор "хороший", то результат измерения не зависит от прибора. Но это справедливо только в том случае, когда все приборы можно прокалибровать единым образом. В классическом случае такая калибровка в принципе возможна. Однако, как показано в [2], для квантовых систем из-за наличия несовместимых измерений такая калибровка не осуществима. Поэтому в квантовом случае результат измерения определяется двумя факторами: элементарным состоянием и типом используемого измерительного прибора. В общем случае будем говорить, что измерительный прибор относится к $\xi$-типу, если в результате измерения наблюдаемой $\hat{A}$ получается значение $\varphi_{\xi}(\hat{A})$.

В некоторых случаях результат измерения может не зависеть от типа измерительного прибора, т.е. $\varphi_{\xi}(\hat{A})=\varphi_{\xi^{\prime}}(\hat{A})$ для всех $\mathfrak{Q}_{\xi}, \mathfrak{Q}_{\xi^{\prime}}$, содержащих $\hat{A}$. В этом случае будем говорить, что элементарное состояние $\varphi$ стабильно на наблюдаемой $\hat{A}$.

Заметим, что в большинстве доказательств невозможности существования локальной физической реальности, определяющей результат измерения, молчаливо предполагалось, что этот результат не зависит от типа измерительного прибора (см., например, [5], [6]).

На пути однозначного предсказания результата измерения в квантовом случае имеется еще одно препятствие. Дело в том, что элементарное состояние системы экспериментально однозначно зафиксировать нельзя. Для его фиксации мы можем использовать только совместимые измерительные приборы. Такие приборы позволяют определить функционал $\varphi_{\xi}(\cdot)$ только для одного значения $\xi(\xi=\eta)$. Все другие функционалы $\varphi_{\xi}(\cdot)$, входящие в элементарное состояние $\left[\varphi_{\xi}\right]$, останутся неопределенными. Образно говоря, элементарное состояние - это голографическое изображение физической системы. С помощью классических измерительных приборов можно получить только плоское изображение. При этом каждое измерение изменяет исходную голографическую картину. Поэтому полное голографическое изображение получить нельзя. 
Объединим все элементарные состояния $\left[\varphi_{\xi}\right]$, имеющие одно и то же сужение на подалгебру $\mathfrak{Q}_{\eta}$, т.е. один и тот же функционал $\varphi_{\eta}$, в класс эквивалентности $\{\varphi\}_{\eta}$. Таким образом, экспериментально можно однозначно зафиксировать только класс эквивалентности, к которому принадлежит интересующее нас элементарное состояние. Если нам известно, что некоторое элементарное состояние $\varphi=\left[\varphi_{\xi}\right]$ принадлежит классу эквивалентности $\{\varphi\}_{\eta}$, то мы однозначно можем предсказать, какой результат получится при измерении наблюдаемой $\hat{A} \in \mathfrak{Q}_{\eta}$ : этим результатом будет $\varphi_{\eta}(\hat{A})$. Если же $\hat{A} \notin \mathfrak{Q}_{\eta}$, то ничего определенного о результате измерения сказать нельзя. Для разных элементарных состояний, принадлежащих $\{\varphi\}_{\eta}$, результаты измерений будут разными. Как раз такими физическими свойствами обладает в стандартной квантовой механике квантовое состояние, фиксируемое определенными значениями наблюдаемых $\hat{A}$ из подалгебры $\mathfrak{Q}_{\eta}$.

Для наблюдаемых $\hat{A} \notin \mathfrak{Q}_{\eta}$ можно предсказать только вероятности получения различных результатов. В стандартном математическом аппарате квантовой механики постулируется, что эти вероятности определяются либо вектором некоторого гильбертова пространства, либо статистическим оператором (матрицей плотности). Этот постулат для практических целей работает очень хорошо, но его обоснование на интуитивном уровне непонятно. Используемый в настоящей статье подход позволяет дать такое обоснование. Естественно считать, что вероятностное распределение результатов измерения определяется вероятностным распределением различных элементарных состояний в классе эквивалентности $\{\varphi\}_{\eta}$. Поэтому примем следующий постулат.

ПостулАТ 5. Класс эквивалентности $\{\varphi\}_{\eta}$ можно оснастить структурой вероятностного пространства.

Заметим, что элементарное состояние удовлетворяет требованиям, которые предъявляет классическая (колмогоровская [7]) теория вероятностей к элементарному событию: в каждом испытании реализуется одно и только одно элементарное событие, т.е. элементарные события являются взаимоисключающими. Это позволяет снабдить $\{\varphi\}_{\eta}$ стандартной (классической) структурой вероятностного пространства $(\Omega, \mathcal{F}, P)$ (см., например, [7], [8]). Здесь $\Omega$ - множество элементарных событий (состояний), $\mathcal{F}$ - булева $\sigma$-алгебра событий $F, P$ - вероятность реализации события $F \in \mathcal{F}$. Событие $F$ - это некоторое подмножество множества $\Omega$. Считается, что в испытании реализуется событие $F$, если реализовалось одно из элементарных событий, принадлежащих этому подмножеству.

Таким образом, для квантовых систем не нужно придумывать какую-то специальную искусственную квантовую теорию вероятностей, а можно воспользоваться стандартной теорией вероятностей. Нужно только учесть [2], [3], что в квантовом случае допустимы не все возможные булевы алгебры $\mathcal{F}$. Если не учитывать этой особенности квантовых систем, то легко получить неравенства Белла [9]-[11], которые противоречат экспериментальным данным.

Если снабдить $\{\varphi\}_{\eta}$ структурой вероятностного пространства, то методами стандартной теории вероятностей легко построить [2] функционал $\Psi_{\eta}(\hat{A})$, который задает 
среднее значение наблюдаемой $\hat{A}$ по классу эквивалентности $\{\varphi\}_{\eta}$. Чтобы этот функционал можно было отождествить с квантовым средним наблюдаемой $\hat{A}$, этот функционал должен быть линейным. Поэтому следует принять следующий постулат.

ПостулАт 6. Вероятностная структура класса эквивалентности $\{\varphi\}_{\eta}$ такова, что функционал $\Psi_{\eta}(\hat{A})$ линеен на алгебре $\mathfrak{A}$.

В этом случае мы можем считать, что функционал $\Psi_{\eta}(\hat{A})$ описывает соответствующее квантовое состояние. Иными словами, в данном подходе можно дать следующее определение.

ОПРЕДЕЛЕНИЕ. Квантовым состоянием $\Psi_{\eta}(\hat{A})$ назовем класс $\{\varphi\}_{\eta} \varphi_{\eta}$-эквивалентных элементарных состояний, стабильных на подалгебре $\mathfrak{Q}_{\eta}$. Этот класс эквивалентности должен допускать описанную структуру вероятностного пространства.

Определенное таким образом квантовое состояние будет чистым [2].

Ранее отмечалось, что результат конкретного измерения наблюдаемой может зависеть от типа измерительного прибора, с помощью которого это измерение осуществлялось. Вместе с тем опыт показывает, что справедливо следующее утверждение.

ПостулАт 7. Среднее значение наблюдаемой по фиксированному квантовому состоянию не зависит от типа измерительного прибора, используемого для его нахождения.

Имея $C^{*}$-алгебру $\mathfrak{A}$ и линейный функционал $\Psi(\cdot)$ на этой алгебре, мы можем с помощью канонической конструкции Гельфанда-Наймарка-Сигала реализовать представление этой алгебры (см., например, [12]) в виде ограниченных линейных операторов в гильбертовом пространстве $\mathfrak{H}$ :

$$
\hat{A} \longleftrightarrow \Pi(\hat{A}), \quad \hat{A} \in \mathfrak{A}, \quad \Pi(\hat{A}) \in \mathfrak{B}(\mathfrak{H}),
$$

где $\mathfrak{B}(\mathfrak{H})$ - множество линейных ограниченных операторов в $\mathfrak{H}$. При этом среднее значение $\langle\hat{A}\rangle$ наблюдаемой $\hat{A}$ по квантовому состоянию $\Psi$ будет представимо в виде математического ожидания оператора $\Pi(\hat{A})$ :

$$
\langle\hat{A}\rangle=\langle\Psi|\Pi(\hat{A})| \Psi\rangle
$$

где $|\Psi\rangle \in \mathfrak{H}$ - соответствующий вектор гильбертова пространства.

Множество физических систем, элементарные состояния которых образуют класс эквивалентности $\{\varphi\}_{\eta}$, назовем квантовым ансамблем. Таким образом, стандартный математический аппарат квантовой механики применим для описания квантовых ансамблей, но не для описания индивидуальной квантовой системы. Адекватной математической характеристикой индивидуальной квантовой системы является элементарное состояние.

Одно и то же элементарное состояние может рассматриваться в качестве элемента различных множеств. Вероятностные характеристики, которые ассоциируются 
с конкретной физической системой, существенным образом зависят от этих множеств. Поэтому обычное в стандартном подходе утверждение "рассматриваемая физическая система находится в данном квантовом состоянии" в предлагаемом подходе следует переформулировать следующим образом: "рассматриваемая физическая система находится в элементарном состоянии, которое принадлежит данному квантовому состоянию". Одновременно это же элементарное состояние можно считать принадлежащим другому квантовому состоянию. Таким образом, квантовое состояние не является вполне объективной характеристикой индивидуальной физической системы.

\section{3. ЗАПУТАННЫЕ СОСТОЯНИЯ}

В процедуре квантовой телепортации центральную роль играют так называемые запутанные состояния. В литературе наиболее часто рассматривают запутанные состояния, характерные для двухчастичной системы, в которой каждая из частиц может находиться в двух ортогональных квантовых состояниях $| \pm\rangle$ :

$$
\begin{aligned}
\left|\Psi^{(-)}\right\rangle_{12} & =\frac{|+\rangle_{1}|-\rangle_{2}-|-\rangle_{1}|+\rangle_{2}}{\sqrt{2}}, \quad\left|\Psi^{(+)}\right\rangle_{12}=\frac{|+\rangle_{1}|-\rangle_{2}+|-\rangle_{1}|+\rangle_{2}}{\sqrt{2}} \\
\left|\Phi^{(-)}\right\rangle_{12} & =\frac{|+\rangle_{1}|+\rangle_{2}-|-\rangle_{1}|-\rangle_{2}}{\sqrt{2}}, \quad\left|\Phi^{(+)}\right\rangle_{12}=\frac{|+\rangle_{1}|+\rangle_{2}+|-\rangle_{1}|-\rangle_{2}}{\sqrt{2}}
\end{aligned}
$$

Эти состояния часто называют состояниями Белла. Их характерной особенностью является следующее. С помощью измерительных приборов можно выделить любое из этих состояний, однако и после такого выделения нельзя однозначно предсказать, в каком из двух возможных квантовых состояний $|+\rangle$ или $|-\rangle$ мы найдем каждую из частиц в последующем измерении. Вместе с тем после измерения квантового состояния одной из частиц состояние другой частицы предсказывается однозначно.

Состояние $\left|\Psi^{(-)}\right\rangle_{12}$ обычно рассматривается при обсуждении парадокса Эйнштейна-Подольского-Розена [13], поэтому его часто называют ЭПР-состоянием. Так, в варианте, предложенном Бомом [14], рассматривается система, состоящая из двух частиц со спином $1 / 2$. Тогда $|+\rangle$ - это квантовое состояние с проекцией спина на ось $z$, равной $+1 / 2$, a $|-\rangle$ - состояние с проекцией, равной $-1 / 2$.

В состоянии $\left|\Psi^{(-)}\right\rangle_{12}$ полный спин $\mathbf{S}=\mathbf{S}_{1}+\mathbf{S}_{2}$ равен нулю. Состояние $\left|\Psi^{(+)}\right\rangle_{12}-$ это состояние с полным спином 1 и проекцией полного спина на ось $z$, равной 0 ; $\left(\left|\Phi^{(+)}\right\rangle_{12}+\left|\Phi^{(-)}\right\rangle_{12}\right) / \sqrt{2}$ - состояние с полным спином 1 и проекцией $+1 ;\left(\left|\Phi^{(+)}\right\rangle_{12}-\right.$ $\left.\left|\Phi^{(-)}\right\rangle_{12}\right) / \sqrt{2}$ - состояние с полным спином 1 и проекцией -1 . Удобно использовать аналогичную терминологию и для других двухуровневых систем, говоря, что состояния $\left|\Psi^{( \pm)}\right\rangle_{12},\left|\Phi^{( \pm)}\right\rangle_{12}$ характеризуются соответствующими значениями наблюдаемых, которые мы будем называть квазиспинами.

Характерной особенностью состояния $\left|\Psi^{(-)}\right\rangle_{12}$ является его сферическая симметрия. Поэтому оно сохраняет свой вид, если вместо проекций на ось $z$ рассматривать проекции на ось $x$ или вообще на произвольное направление $\mathbf{n}$. В этом состоянии для любого $\mathbf{n}$ справедливо

$$
S_{\mathbf{n} 1}+S_{\mathbf{n} 2}=0,
$$


где $S_{\mathbf{n} i}$ - проекция (квази)спина $i$-й частицы на направление $\mathbf{n}(i=1,2)$.

Для такой системы парадокс ЭПР состоит в следующем. По стандартной идеологии квантовой механики в состоянии $\left|\Psi^{(-)}\right\rangle_{12}$ ни одна из частиц не имеет определенного значения проекции (квази)спина на направление $\mathbf{n}$. Как только мы измеряем такую проекцию у первой частицы, согласно проекционному принципу квантовое состояние системы преобразуется таким образом, что как первая, так и вторая частица приобретают определенные значения (противоположного знака) проекции (квази)спина на направление $\mathbf{n}$. То, что первая частица в результате воздействия измерительного прибора приобрела определенное значение проекции, выглядит вполне правдоподобно. Но очень трудно представить, как измерительный прибор мог повлиять на частицу, если частица и прибор разделены пространственноподобным интервалом.

В рамках схемы, описанной в предыдущем разделе, парадокс ЭПР разрешается тривиально. Квантовое состояние $\left|\Psi^{(-)}\right\rangle_{12}$ описывает не одну двухчастичную систему, а ансамбль таких систем. Пусть индекс $\alpha$ отличает один элемент ансамбля от другого. Каждая из частиц, входящая в $\alpha$-ю систему, находится во вполне определенном элементарном состоянии $\left[\varphi_{\xi 1}^{\alpha}\right]$ для первой частицы и $\left[\varphi_{\xi 2}^{\alpha}\right]$ для второй. В качестве индекса $\xi$, фиксирующего коммутативную подалгебру, в данном случае удобно взять единичный трехмерный вектор $\mathbf{n}$. При этом следует считать, что векторам $\mathbf{n}$ и $\mathbf{- n}$ соответствует одна и та же подалгебра. Тогда характеры $\varphi_{\xi 1}^{\alpha}$ и $\varphi_{\xi 2}^{\alpha}$, входящие в элементарные состояния, описывают проекции (квази)спина на направление $\mathbf{n}$ для первой и второй частицы. Они принимают следующие значения [2]: $\varphi_{\mathbf{n} i}^{\alpha}=S_{\mathbf{n} i}^{\alpha}$, где $S_{\mathbf{n} i}^{\alpha}=-S_{-\mathbf{n} i}^{\alpha}(i=1,2)$ и $S_{\mathbf{n} 1}^{\alpha}=-S_{\mathbf{n} 2}^{\alpha}$, а $S_{\mathbf{n} 1}^{\alpha}$ в зависимости от $\alpha$ и $\mathbf{n}$ равно либо $+1 / 2$, либо $-1 / 2$.

При выполнении таких условий элементарное состояние одной частицы ЭПР-пары является негативной копией элементарного состояния другой частицы. Поэтому измерение значения некоторой наблюдаемой у первой частицы автоматически является измерением соответствующей наблюдаемой у второй частицы вне зависимости от того, где эта частица локализована. Такое измерение называется косвенным. При нем измеряемый объект не подвергается воздействию измерительного прибора. Поэтому его элементарное состояние не меняется. Жесткая корреляция между элементарными состояниями частиц в ЭПР-паре является ее характерной особенностью. Эта корреляция возникает при приготовлении ЭПР-пары, а не при последующем измерении. Корреляция не является следствием проекционного принципа, скорее наоборот, утверждения, которые содержатся в проекционном принципе, являются следствием такой корреляции. Аналогичные корреляции присущи и другим запутанным состояниям.

\section{4. НЕКОТОРЫЕ ПРИБОРЫ КВАНТОВОЙ ФИЗИКИ}

Для понимания процедуры квантовой телепортации необходимо понимание принципа работы используемых приборов. В этом разделе будет дано очень краткое описание трех таких приборов. 
В предыдущем разделе парадокс ЭПР был рассмотрен на примере системы, состоящей из двух частиц со спинами $1 / 2$. Однако технически весьма сложно для такой системы создать требуемую ЭПР-пару. Значительно проще аналогичную пару получить оптическими средствами. Соответствующий прибор называется параметрическим преобразователем частоты вниз второго рода. В русской литературе этот прибор часто называют источником спонтанного параметрического рассеяния (СПР-источником). Основой СПР-источника является нелинейный оптический кристалл, который облучается ультрафиолетовым лазером. Лазерные фотоны рассеиваются в кристалле. Как правило, при таком рассеянии один фотон на входе дает один фотон на выходе. Однако иногда с гораздо меньшей (на много порядков) интенсивностью один фотон порождает два фотона. При СПР второго типа эти фотоны оказываются поляризованными в двух взаимно ортогональных направлениях $H$ (горизонтальном) и $V$ (вертикальном). Можно подобрать такие условия, что эти фотоны будут образовывать ЭПР-пару, т.е. их квантовое состояние можно описать вектором $\left|\Psi^{(-)}\right\rangle_{12}$ (см. формулу (1)), где $|+\rangle$ соответствует горизонтальной поляризации, а $|-\rangle-$ вертикальной.

Второй прибор - это (простой) светоделитель. Он предназначен для перемешивания двух пучков фотонов без изменения их внутренних характеристик. Наглядно этот прибор можно представить в виде полупрозрачной пластины (см. рис. 1), на которую сверху и снизу в одной плоскости и под одинаковыми углами падают два пучка фотонов. Если фотоны из разных пучков попадают в пластину в разные моменты времени, то каждый из них независимо с вероятностью $1 / 2$ проходит сквозь пластину или с вероятностью $1 / 2$ от нее отражается. Если же два фотона попадают одновременно, то они интерферируют между собой согласно формуле

$$
\begin{aligned}
|H, V\rangle_{\mathrm{u}}^{\text {in }} & \rightarrow \frac{|H, V\rangle_{\mathrm{u}}^{\text {out }}+|H, V\rangle_{\mathrm{d}}^{\text {out }}}{\sqrt{2}}, \\
|H, V\rangle_{\mathrm{d}}^{\text {in }} & \rightarrow \frac{|H, V\rangle_{\mathrm{u}}^{\text {out }}-|H, V\rangle_{\mathrm{d}}^{\text {out }}}{\sqrt{2}},
\end{aligned}
$$

гду индекс и обозначает верхний пучок, d - нижний пучок, $|H, V\rangle$ означает либо $|H\rangle$, либо $|V\rangle$.

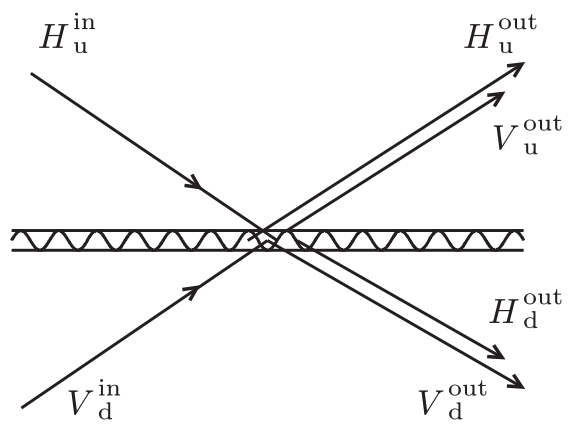

Рис. 1. Простой светоделитель. 
На рис. 1 для определенности изображен случай, когда падающий верхний пучок имеет горизонтальную поляризацию (описывается вектором $|H\rangle_{\mathrm{u}}^{\mathrm{in}}$ ), а нижний вертикальную $|V\rangle_{\mathrm{d}}^{\text {in }}$.

Из формул (2) следует, что при прохождении светоделителя состояния Белла (см. формулы (1)) преобразуются следующим образом:

$$
\begin{aligned}
\left|\Psi^{(-)}\right\rangle_{\mathrm{ud}}^{\text {in }} & \rightarrow \frac{|H\rangle_{\mathrm{u}}^{\text {out }}|V\rangle_{\mathrm{d}}^{\text {out }}-|V\rangle_{\mathrm{u}}^{\text {out }}|H\rangle_{\mathrm{d}}^{\text {out }}}{\sqrt{2}} \equiv-\left|\Psi^{(-)}\right\rangle_{\mathrm{ud}}^{\text {out }}, \\
\left|\Psi^{(+)}\right\rangle_{\mathrm{ud}}^{\text {in }} & \rightarrow \frac{|H\rangle_{\mathrm{u}}^{\text {out }}|V\rangle_{\mathrm{u}}^{\text {out }}-|H\rangle_{\mathrm{d}}^{\text {out }}|V\rangle_{\mathrm{d}}^{\text {out }}}{\sqrt{2}}, \\
\left|\Phi^{(-)}\right\rangle_{\mathrm{ud}}^{\text {in }} & \rightarrow \frac{|H\rangle_{\mathrm{u}}^{\text {out }}|H\rangle_{\mathrm{u}}^{\text {out }}-|V\rangle_{\mathrm{u}}^{\text {out }}|V\rangle_{\mathrm{u}}^{\text {out }}-|H\rangle_{\mathrm{d}}^{\text {out }}|H\rangle_{\mathrm{d}}^{\text {out }}+|V\rangle_{\mathrm{d}}^{\text {out }}|V\rangle_{\mathrm{d}}^{\text {out }}}{\sqrt{2}}, \\
\left|\Phi^{(+)}\right\rangle_{\mathrm{ud}}^{\text {in }} & \rightarrow \frac{|H\rangle_{\mathrm{u}}^{\text {out }}|H\rangle_{\mathrm{u}}^{\text {out }}+|V\rangle_{\mathrm{u}}^{\text {out }}|V\rangle_{\mathrm{u}}^{\text {out }}-|H\rangle_{\mathrm{d}}^{\text {out }}|H\rangle_{\mathrm{d}}^{\text {out }}-|V\rangle_{\mathrm{d}}^{\text {out }}|V\rangle_{\mathrm{d}}^{\text {out }}}{\sqrt{2}} .
\end{aligned}
$$

Другими словами, при падающем состоянии $\left|\Psi^{(-)}\right\rangle$in (квазиспин 0) после светоделителя фотоны в каждой одновременной паре оказываются по разные стороны от пластины, а при состояниях $\left.\left|\Psi^{(+)}\right\rangle\right\rangle_{\mathrm{ud}}^{\text {in }},\left|\Phi^{(-)}\right\rangle_{\mathrm{ud}}^{\text {in }},\left|\Phi^{(+)}\right\rangle_{\mathrm{ud}}^{\text {in }}$ (квазиспин 1) - по одну сторону.

Третий прибор - это поляризационный светоделитель (ПСД). Геометрия этого прибора определяет так называемый (ортогональный) поляризационный базис. Пучок фотонов, падающий на ПСД, направляется по первому базисному вектору. Два других базисных вектора определяют вертикальное и горизонтальное направления. Если пучок поляризован по горизонтальному направлению, то он проходит ПСД насквозь, если по вертикальному - отражается. Часто ПСД используется в сочетании с детекторами, которые располагаются в направлениях распространения вторичных пучков. Такое устройство позволяет определить поляризацию фотонов.

Если ПСД повернуть вокруг первого базисного вектора на некоторый угол, то мы получим новый поляризационный базис. Пучок, имевший определенную поляризацию в исходном базисе, после прохождения ПСД разложится на два подпучка, каждый из которых будет иметь определенную (горизонтальную или вертикальную) поляризацию в новом базисе. Если используемые детекторы однофотонные (реагирующие на отдельные фотоны), то можно определить, какую поляризацию имеет отдельный фотон в новом базисе. По стандартной идеологии квантовой механики отдельный фотон пучка, поляризованного вдоль одного из векторов исходного базиca, не имеет определенной поляризации относительно векторов повернутого базиса. Такую поляризацию он приобретает при прохождении ПСД. При этом такой процесс селекции не имеет никакой объективной причины. В этом смысле он является чисто случайным.

По идеологии, изложенной в разделе 2 , каждый отдельный фотон имеет определенную поляризацию (вертикальную или горизонтальную) в любом поляризационном базисе. Все эти поляризации определяются элементарным состоянием данного 
фотона. Однако изначально нам известна поляризация только в исходном базисе. Повернутый ПСД сортирует фотоны исходного пучка по поляризациям в своем поляризационном базисе. Одновременно с этим ПСД неконтролируемым образом возмущает известную ранее поляризацию в исходном базисе. Таким образом, с помощью ПСД и однофотонных детекторов можно определить поляризацию каждого фотона по любому направлению, но только по одному.

\section{5. КВАНТОВАЯ ТЕЛЕПОРТАЦИЯ}

На рис. 2 изображена схема квантовой телепортации. Здесь $\mathrm{S}$ - источник начального состояния; $\mathrm{EPR}$ - источник ЭПР-пар, $A$ - анализатор состояний Белла (Алиса), $B$ - унитарный преобразователь (Боб), $\{\mathrm{C}\}$ - канал классической связи, $\{1\}$ - носитель начального телепортируемого состояния, $\{2\}-\{3\}-$ ЭПР-пара, $\{4\}-$ носитель конечного телепортируемого состояния.

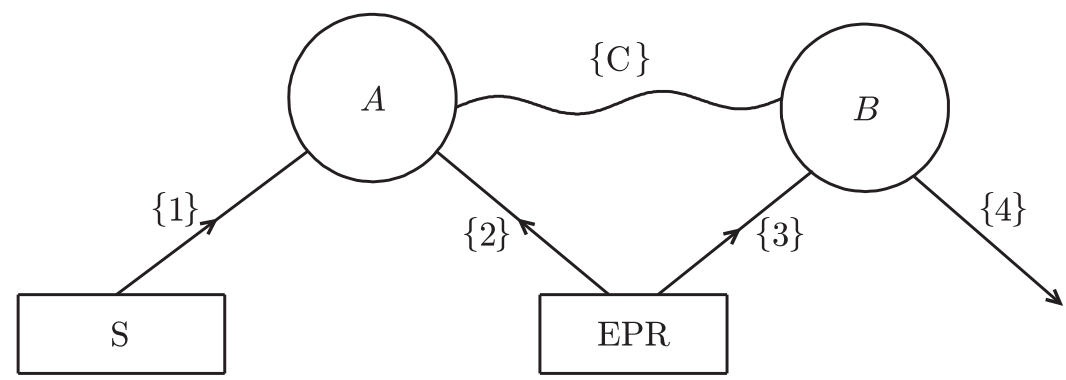

Рис. 2. Схема квантовой телепортации.

Приведем стандартное описание схемы телепортации (см., например, [15]). Каждая из частиц $\{1\},\{2\},\{3\},\{4\}$, участвующих в процессе, может находиться на одном из квантовых уровней $|+\rangle$ или $|-\rangle$. Источник $\mathrm{S}$ испускает частицу $\{1\}$ в квантовом состоянии $|\Psi\rangle_{1}=\alpha|+\rangle+\beta|-\rangle$, где $|\alpha|^{2}+|\beta|^{2}=1$. В общем случае $\alpha$ и $\beta$ могут быть неизвестны. ЭПР-источник испускает частицы $\{2\}$ и $\{3\}$ в квантовом состоянии $\left|\Psi^{(-)}\right\rangle_{23}$ (см. формулу (1)). Квантовое состояние трехчастичной системы, состоящей из частиц $\{1\},\{2\},\{3\}$, описывается вектором $|\Psi\rangle_{123}=|\Psi\rangle_{1} \otimes\left|\Psi^{(-)}\right\rangle_{23}$, который можно представить в виде разложения по состояниям Белла частиц $\{1\}$ и $\{2\}$ :

$$
\begin{aligned}
|\Psi\rangle_{123}= & \frac{1}{2}\left\{\left|\Psi^{(-)}\right\rangle_{12}\left(-\alpha|+\rangle_{3}-\beta|-\rangle_{3}\right)+\left|\Psi^{(+)}\right\rangle_{12}\left(-\alpha|+\rangle_{3}+\beta|-\rangle_{3}\right)+\right. \\
& \left.+\left|\Phi^{(-)}\right\rangle_{12}\left(\alpha|-\rangle_{3}+\beta|+\rangle_{3}\right)+\left|\Phi^{(+)}\right\rangle_{12}\left(\alpha|-\rangle_{3}-\beta|+\rangle_{3}\right)\right\} .
\end{aligned}
$$

Алиса с помощью анализатора $A$ проводит измерение, для того чтобы найти, в каком из четырех состояний Белла находятся доступные ей частицы $\{1\}$ и $\{2\}$. 
Пусть, например, она получает результат $\left|\Psi^{(-)}\right\rangle_{12}$. Тогда согласно проекционному принципу после такого измерения трехчастичная система коллапсирует в состояние $\left|\Psi^{\prime}\right\rangle_{123}=\left|\Psi^{(-)}\right\rangle_{12}\left(-\alpha|+\rangle_{3}-\beta|-\rangle_{3}\right)$. По каналу классической связи Алиса сообщает, что она обнаружила частицы $\{1\}$ и $\{2\}$ в состоянии $\left|\Psi^{(-)}\right\rangle_{12}$. Боб, ничего не делая, пропускает частицу $\{3\}$ дальше. Эта частица будет в состоянии $|\Psi\rangle_{4}=\left(-\alpha|+\rangle_{3}-\beta|-\rangle_{3}\right)$, которое совпадает с состоянием $|\Psi\rangle_{1}$. Если Алиса находит другое состояние Белла, то она по классическому каналу связи сообщает Бобу, какое из трех унитарных преобразований $-|+\rangle \longrightarrow|+\rangle,|+\rangle \longleftrightarrow|-\rangle$, $-|+\rangle \longrightarrow|+\rangle \longleftrightarrow|-\rangle$ он должен совершить над частицей $\{3\}$, чтобы получить состояние $|\Psi\rangle_{4}$, совпадающее с $|\Psi\rangle_{1}$.

В таком описании процесса телепортации особенно загадочным выглядит случай, когда Алиса обнаруживает результат $\left|\Psi^{(-)}\right\rangle_{12}$. До измерения между частицами $\{1\}$ и $\{3\}$ не было никакой корреляции. Очень трудно представить, как, физически не воздействуя на частицу $\{3\}$, Алиса смогла заставить ее перейти в то квантовое состояние, в котором находилась частица $\{1\}$. При этом Алиса даже не знала, какое это состояние.

Посмотрим теперь, как тот же процесс телепортации можно описать, используя понятие элементарного состояния. В этом случае квантовому состоянию $|\Psi\rangle_{1}$ соответствует целый пучок частиц $\{1\}$, которые находятся в различных элементарных состояниях, но все эти состояния принадлежат одному классу эквивалентности. Соответственно в эксперименте потребуется не одна ЭПР-пара $\{2\}-\{3\}$, а пучок таких пар. Фигурирующие в квантовом состоянии $|\Psi\rangle_{1}$ числа $\alpha$ и $\beta$ задают направление $\mathbf{n}$, вдоль которого проекция квазиспина каждой частицы пучка $\{1\}$ с определенностью равна $1 / 2$. Направим ось $z$ по направлению $\mathbf{n}$. Тогда в квантовом состоянии $-\alpha|+\rangle_{3}-\beta|-\rangle_{3}$ для проекции квазиспина будет выполняться равенство $S_{z}=+1 / 2$, в состоянии $-\alpha|+\rangle_{3}+\beta|-\rangle_{3}-$ равенство $S_{z}=-1 / 2$, в состоянии $\alpha|-\rangle_{3}+\beta|+\rangle_{3}-$ равенство $S_{x}=+1 / 2$, в состоянии $\left.|\alpha|-\right\rangle_{3}-\beta|+\rangle_{3}-$ равенство $S_{x}=-1 / 2$.

Будем теперь рассматривать в качестве измерительного прибора анализатор $A$ совместно с частицей $\{1\}$. Действие этого комбинированного измерительного прибора на пучок частиц $\{2\}$ можно интерпретировать двумя способами (см. формулу (4)). С одной стороны, этот прибор разделяет пучок частиц $\{2\}$ на четыре подпучка, в каждом из которых частицы $\{2\}$ (совместно с частицами $\{1\}$ ) находятся в одном из состояний Белла. Этот результат фиксируется анализатором $A$. С другой стороны, в каждом из этих четырех подпучков частицы $\{2\}$ будут иметь определенные значения проекций квазиспина либо на ось $z$, либо на ось $x$. Благодаря жесткой корреляции элементарных состояний частиц $\{2\}$ и $\{3\}$ при этом автоматически произойдет разбиение пучка частиц $\{3\}$ на четыре подпучка, в каждом из которых частицы $\{3\}$ будут иметь определенные проекции квазиспина. То есть в данном случае мы имеем типичный пример косвенного измерения проекции квазиспина у частицы $\{3\}$. По классическому каналу связи Алиса сообщает Бобу результат такого косвенного измерения. В соответствии с этой информацией Боб применяет к частицам $\{3\}$ то или иное унитарное преобразование. Измерение, производимое 
Алисой, никак не влияет на элементарное состояние частицы $\{3\}$. В результате этого измерения приобретается только некоторая информация об этом элементарном состоянии, необходимая для дальнейших действий Боба.

Обратим внимание на то, что после всех описанных манипуляций элементарное состояние частицы $\{3\}$ не становится таким же, как было у частицы $\{1\}$. Эти частицы только оказываются в одном классе эквивалентности. Таким образом, частица $\{3\}$ не становится точной копией частицы $\{1\}$, поэтому термин “телепортация" для описания этой процедуры не является особенно удачным.

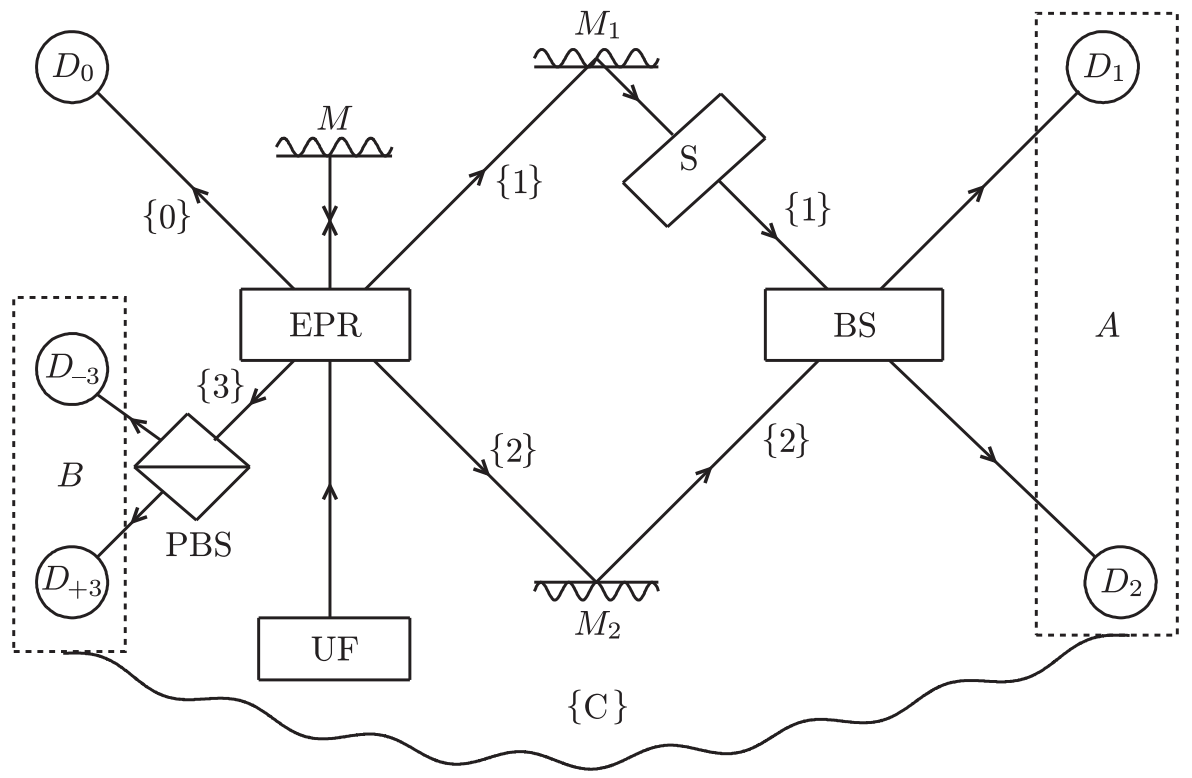

Рис. 3. Принципиальная схема эксперимента по квантовой телепортации.

Теперь обсудим реальный эксперимент, в котором наблюдалась телепортация [16]. Принципиальная схема экспериментальной установки изображена на рис. 3. Здесь $\mathrm{UF}$ - лазер (источник ультрафиолетового импульса), $\mathrm{EPR}$ - ЭПР-источник; $M, M_{1}$, $M_{2}$ - полностью отражающие зеркала, BS - простой светоделитель, PBS - поляризационный светоделитель, $\mathrm{S}$ - кодировщик начального состояния фотона $\{1\}$; $D_{0}, D_{1}, D_{2}, D_{+3}, D_{-3}$ - детекторы, $\{\mathrm{C}\}$ - классический канал связи (схема совпадений для комбинаций детекторов).

Далее для простоты рассуждений мы будем считать, что все рассматриваемые фотоны распространяются в одной плоскости, и горизонтальное направление исходного поляризационного базиса лежит в этой плоскости.

Лазерный ультрафиолетовый импульс попадает в нелинейный кристалл EPR. B кристалле рождается ЭПР-пара $\{0\}-\{1\}$. После прохождения кристалла импульс отражается от зеркала $M$ и вновь попадает в кристалл, где рождает вторую ЭПР-пару $\{2\}-\{3\}$. Фотон $\{1\}$, отражаясь от зеркала $M_{1}$, попадает в кодировщик S, где он 
приобретает определенную поляризацию. В реальном эксперименте изучались два варианта: поляризация под углом в $45^{\circ}$ и под углом в $90^{\circ}$. После этого фотон $\{1\}$ попадает в светоделитель BS.

Фотон $\{2\}$ после отражения от зеркала $M_{2}$ попадает в тот же светоделитель BS, но с другой стороны. С помощью передвижения зеркала $M$ можно добиться того, чтобы фотоны $\{1\}$ и $\{2\}$ попадали в светоделитель BS одновременно. Тогда в светоделителе они интерферируют и после светоделителя либо оба попадают в один из детекторов $D_{1}$ или $D_{2}$, либо один из них попадает в детектор $D_{1}$, а другой в детектор $D_{2}$.

Фотон $\{3\}$ направляется в поляризационный светоделитель, после которого в зависимости от поляризации попадает либо в детектор $D_{+3}$, либо в детектор $D_{-3}$. Фотон $\{0\}$ сразу направляется в детектор $D_{0}$. Схема совпадений $\{\mathrm{C}\}$ регистрирует события, в которых одновременно срабатывают либо детекторы $D_{0}, D_{1}, D_{2}, D_{+3}$, либо детекторы $D_{0}, D_{1}, D_{2}, D_{-3}$.

При проведении эксперимента учитывалось, что рождение ЭПР-пары - это очень редкое событие на фоне рождения одиночных фотонов, а рождение двух ЭПР-пар еще гораздо более редкое событие. Поэтому очень важно отделить случайные совпадения в работе детекторов от значимых совпадений. В этом плане детектор $D_{0}$ нужен для того, чтобы выделить события, в которых действительно присутствует фотон $\{1\}$, состояние которого подлежит телепортации. Совпадение в работе детекторов $D_{1}$ и $D_{2}$ авторами эксперимента интерпретировалось как свидетельство того, что после такого измерения фотоны $\{1\}$ и $\{2\}$ оказывались в квантовом состоянии $\left|\Psi^{(-)}\right\rangle_{12}$. Соответственно, фотон $\{3\}$ приобретал то же квантовое состояние, что и фотон $\{1\}$ после прохождения кодировщика S. Для проверки этого факта поляризационный светоделитель PBS ориентировался так, что если фотон $\{3\}$ обладал той же поляризацией, которая была закодирована в фотоне $\{1\}$, то фотон $\{3\}$ должен был попасть в детектор $D_{+3}$. Поэтому факту телепортации должна соответствовать ситуация, в которой наблюдается совпадение $D_{0}-D_{1}-D_{2}-D_{+3}$ и отсутствует совпадение $D_{0}-D_{1}-D_{2}-D_{-3}$.

Реально в эксперименте исследовалась частота такого типа совпадений в зависимости от положения зеркала $M$, т.е. в зависимости от того, насколько одновременно попадают фотоны $\{1\}$ и $\{2\}$ в светоделитель BS. Иными словами, интерферируют ли они в светоделителе или рассеиваются независимо друг от друга. При полной интерференции число совпадений $D_{0}-D_{1}-D_{2}-D_{-3}$ должно спадать до нуля. При отсутствии интерференции оба типа совпадений оказываются случайными, и их частота становится одинаковой. Действительно, в графике зависимости частоты совпадений $D_{0}-D_{1}-D_{2}-D_{-3}$ от положения зеркала $M$ наблюдался значительный провал (примерно на один порядок), который соответствовал интерференции фотонов $\{1\}$ и $\{2\}$. В последующих аналогичных экспериментах [17] этот факт получил подтверждение. Однако был получен и новый трудно интерпретируемый результат. Дело в том, что за счет изменения длин путей фотонов $\{1\}$ и $\{2\}$ можно добиться того, что описанный эффект достигается в том случае, когда в схему совпадений вносится 
временна́я задержка для детекторов $D_{1}$ и $D_{2}$. В этом случае факт телепортации, регистрируемый детекторами $D_{+3}$ и $D_{-3}$, получается раньше, чем эта телепортация произошла в результате работы детекторов $D_{1}$ и $D_{2}$.

Такой результат действительно наблюдался. Более того, в принципе, можно добиться того, что факт телепортации будет зафиксирован раньше, чем фотон $\{1\}$ приобретет определенную поляризацию. Строго говоря, все это формально не противоречит стандартному математическому аппарату квантовой механики, но как-то трудно поверить, что будущее может влиять на прошлое.

Посмотрим теперь, как тот же эксперимент можно интерпретировать в рамках схемы, описанной в разделе 2. Сначала рассмотрим случай, когда фотон $\{1\}$ после прохождения кодировщика приобретает вертикальную поляризацию в исходном базисе. Пусть поляризационный светоделитель PBS повернут относительно исходного базиса на $90^{\circ}$. Тогда, если фотон $\{3\}$ в исходном базисе поляризован вертикально, то он попадет в детектор $D_{+3}$, а если горизонтально, то в детектор $D_{-3}$.

Рассмотрим случай, когда срабатывает детектор $D_{-3}$. Так как фотоны $\{2\}$ и $\{3\}$ образуют ЭПР-пару, фотон $\{2\}$ при этом имеет вертикальную поляризацию. Таким образом, в этом случае оба фотона $\{1\}$ и $\{2\}$, попадающие в светоделитель $\mathrm{BS}$, имеют одинаковую поляризацию. Поэтому если они оказываются в светоделителе BS одновременно, то после светоделителя (см. формулу (3)) они оба попадут в один детектор (в $D_{1}$ или в $D_{2}$ ). То есть теоретически вероятность совпадения $D_{0}-D_{1}-D_{2}-D_{-3}$ равна нулю. В действительности эта вероятность будет отлична от нуля хотя бы потому, что ЭПР-источник не создает идеальные пары.

В отличие от стандартной квантовой механики, в данной схеме предсказывается жесткая временна́я корреляция событий. В пределах малой ошибки, связанной с длительностью лазерного импульса и возможной неодновременностью испускания фотонов $\{2\}$ и $\{3\}$, положительный эффект будет достигаться, если схема совпадений отрегулирована следующим образом. Для нее должны считаться одновременными моменты времени $t_{0}$ (время, когда фотон $\{0\}$ достигает детектора $\left.D_{0}\right), t_{12}$ (время, когда фотоны $\{1\}$ и $\{2\}$ достигают детекторов $D_{1}$ и $D_{2}$ ) и $t_{3}$ (время, когда фотон $\{3\}$ достигает детектора $D_{-3}$ или $\left.D_{+3}\right)$. Астрономически времена $t_{0}, t_{12}, t_{3}$ могут находиться в любых соотношениях.

Рассмотрим теперь случай, когда в исходном поляризационном базисе фотон $\{1\}$ поляризован под углом $45^{\circ}$. Пусть детектор $D_{-3}$ регистрирует фотон, поляризованный под углом $-45^{\circ}$. Тогда фотон $\{2\}$ будет поляризован под углом $45^{\circ}$. Дальнейшие рассуждения легче проводить не терминах поляризации, а в терминах квазспина. Поляризации в $45^{\circ}$ сопоставим ориентацию квазиспина по оси $x$, т.е. $\left|45^{\circ}\right\rangle \rightarrow\left|S_{x}=1 / 2\right\rangle$. Таким образом, в рассматриваемом случае элементарное состояние двухчастичной системы $\{12\}$, состоящей из фотонов $\{1\}$ и $\{2\}$, будет принадлежать квантовому состоянию $\left|S_{x}=1 / 2\right\rangle_{1}\left|S_{x}=1 / 2\right\rangle_{2}$. По стандартным правилам квантовой механики в этом квантовом состоянии полный квазиспин равняется 1. Поэтому согласно формулам (3) оба фотона после прохождения светоделителя $\mathrm{BS}$ должны попасть в один и тот же детектор $\left(D_{1}\right.$ или $\left.D_{2}\right)$. В результате схема 
совпадений этот случай не зарегистрирует, т.е. результат будет такой же, как при поляризации фотона под углом $90^{\circ}$.

Однако в действительности ситуация может оказаться более сложной. Дело в том, что двухчастичная система $\{12\}$ не приготавливалась как квантовая система со значением квадрата полного квазиспина $S^{2}$, равным 2. Отбора элементарных состояний двухчастичной системы $\{12\}$ по значению наблюдаемой $\widehat{S}^{2}$ не было. Поэтому нет гарантии, что в каждом элементарном событии такое значение обязательно будет. В лучшем случае, если верить постулату 7, можно только утверждать, что среднее значение наблюдаемой $\widehat{S}^{2}$ по бесконечному числу испытаний равняется 2 .

В реальном эксперименте число случаев, когда регистрируется наличие совпадений $D_{0}-D_{1}-D_{2}-D_{+3}$ и отсутствие совпадений $D_{0}-D_{1}-D_{2}-D_{-3}$, очень невелико. Поэтому в каждом испытании вероятность отклонения значения наблюдаемой $\widehat{S}^{2}$ от среднего значения достаточно велика. При улучшении статистики эта вероятность должна уменьшаться. Отсюда вроде бы следует, что улучшение статистики бесполезно при попытке обнаружить отклонение от предсказаний стандартной квантовой механики. Однако это не так. Нужно только относиться к этим отклонениям не как к ошибкам измерения, а как к изучаемому эффекту.

Можно, например, изучать величину

$$
\rho=\frac{N_{-}(45)}{N_{-}(90)} \quad \text { при } \quad N_{-}(45)+N_{+}(45)=N_{-}(90)+N_{+}(90),
$$

в которой $N_{-}(45)$ - количество совпадений $D_{0}-D_{1}-D_{2}-D_{-3}$ в отсутствие совпадений $D_{0}-D_{1}-D_{2}-D_{+3}$ и $N_{+}(45)$ - количество совпадений $D_{0}-D_{1}-D_{2}-D_{+3}$ в отсутствие совпадений $D_{0}-D_{1}-D_{2}-D_{-3}$ при поляризации фотона $\{1\}$ под углом в $45^{\circ}$, а $N_{-}(90)$ и $N_{+}(90)$ - те же числа при поляризации под углом в $90^{\circ}$. Если в рассматриваемом случае использовать стандартную квантовую механику, то следует ожидать, что при улучшении статистики величина $\rho$ должна стремиться к единице. Если же использовать предлагаемый в данной статье подход, то следует ожидать, что величина $\rho$ всегда будет больше единицы.

Следует сказать, что подобный результат экспериментально уже обнаружен [17]. Однако авторы эксперимента склонны рассматривать этот факт как экспериментальную ошибку. В такой ситуации следовало бы провести специальный эксперимент с целью досконального исследования этого эффекта.

\section{6. ТЕЛЕПОРТАЦИЯ ЗАПУТАННОСТИ}

Наиболее сильным аргументом в пользу нелокальности, присущей квантовым измерениям, часто считается явление, которое получило название "телепортация запутанности". Суть этого явления можно понять из рис. 4. На этом рисунке $\mathrm{EPR}_{1}$ и $\mathrm{EPR}_{2}$ - источники независимых ЭПР-пар, $A$ - анализатор состояний Белла (Алиса); $B$ - унитарный преобразователь (Боб), $\{\mathrm{C}\}$ - классический канал связи (схема совпадений,$\{0\}-\{1\}-$ первая ЭПР-пара, $\{2\}-\{3\}-$ вторая ЭПР-пара. 


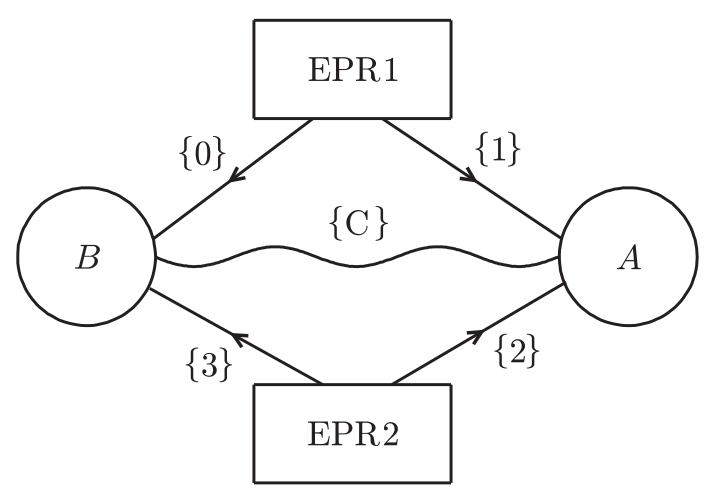

Рис. 4. Схема телепортации запутанности.

Опять сначала приведем стандартное описание схемы телепортации запутанности [18]. Источник $\mathrm{EPR}_{1}$ испускает пару $\{0\}-\{1\}$ в квантовом состоянии $\left|\Psi^{(-)}\right\rangle_{01}$. Источник $\mathrm{EPR}_{2}$ независимо испускает пару $\{2\}-\{3\}$ в квантовом состоянии $\left|\Psi^{(-)}\right\rangle_{23}$. Вектор четырехчастичного квантового состояния $|\Psi\rangle_{0123}=\left|\Psi^{(-)}\right\rangle_{01} \otimes\left|\Psi^{(-)}\right\rangle_{23}$ может быть представлен в виде разложения по состояниям Белла частиц $\{1\}-\{2\}$ :

$|\Psi\rangle_{0123}=\frac{1}{2}\left\{\left|\Psi^{(+)}\right\rangle_{03}\left|\Psi^{(+)}\right\rangle_{12}-\left|\Psi^{(-)}\right\rangle_{03}\left|\Psi^{(-)}\right\rangle_{12}-\left|\Phi^{(+)}\right\rangle_{03}\left|\Phi^{(+)}\right\rangle_{12}+\left|\Phi^{(-)}\right\rangle_{03}\left|\Psi^{(-)}\right\rangle_{12}\right\}$

Одновременно эта формула является разложением вектора $|\Psi\rangle_{0123}$ по состояниям Белла частиц $\{0\}-\{3\}$. Алиса с помощью анализатора $A$ определяет, в каком из четырех состояний Белла находится пара $\{1\}-\{2\}$ и сообщает результат Бобу по классическому каналу связи. Пусть, например, таким состоянием оказывается $\left|\Psi^{(-)}\right\rangle_{12}$. Тогда согласно проекционному принципу состояние $|\Psi\rangle_{0123}$ коллапсирует в состояние $\left|\Psi^{(-)}\right\rangle_{03}\left|\Psi^{(-)}\right\rangle_{12}$. Поэтому теперь Боб может утверждать, что пара $\{0\}-\{3\}$ находится в квантовом запутанном состоянии $\left|\Psi^{(-)}\right\rangle_{03}$.

Первоначально частицы $\{0\}$ и $\{3\}$ были совершенно независимы. В дальнейшем они никакому физическому воздействию не подвергались. Все манипуляции проводились с частицами $\{1\}$ и $\{2\}$. Тем не менее после этих манипуляций частицы $\{0\}$ и $\{3\}$ каким-то таинственным образом оказались в запутанном состоянии. В рамках подхода, принятого в настоящей статье, можно дать гораздо более наглядное описание корреляции между частицами $\{0\}$ и $\{3\}$.

Алиса с помощью анализатора $A$ разделяет пучок частиц $\{1\}$ и $\{2\}$ на четыре подпучка по признаку наличия в каждом подпучке определенной корреляции между частицами. Эти корреляции не создаются анализатором $A$, анализатор $A$ служит только для выделения тех пар, в которых такие корреляции возникли случайно при испускании этих частиц источниками $\mathrm{EPR}_{1}$ и $\mathrm{EPR}_{2}$.

Элементарное состояние частицы $\{0\}$ жестко коррелировано с элементарным состоянием частицы $\{1\}$ и аналогично для частиц $\{2\}$ и $\{3\}$, поэтому разделение на 
подпучки пучка частиц $\{1\}$ и $\{2\}$ автоматически приводит к разделению на соответствующие подпучки пучка частиц $\{0\}$ и $\{3\}$. В каждой паре $\{0\}-\{3\}$ будет иметь место корреляция, которая является (негативной) копией корреляции в паре $\{1\}-\{2\}$. Классический канал связи $\{\mathrm{C}\}$ позволяет установить, к какому из подпучков принадлежит конкретная пара $\{0\}-\{3\}$. Здесь надо иметь в виду, что корреляция в паре $\{0\}-\{3\}$ является копией той корреляции в паре $\{1\}-\{2\}$, которая была до анализатора $A$. Вместе с тем анализатор $A$ может выполнять функцию не только измерительного прибора, но и прибора, приготавливающего новое элементарное состояние. Поэтому корреляция в паре $\{0\}-\{3\}$ в общем случае не будет точной копией корреляции в паре $\{1\}-\{2\}$ после прохождения последней анализатора $A$.

Далее обсудим реальные эксперименты, в которых изучалось явление телепортации запутанности [17], [18]. Принципиальная схема экспериментальной установки изображена на рис. 5. В основном она аналогична установке, изображенной на рис. 3. Разница в том, что на рис. 5 отсутствует кодировщик начального состояния, зато вместо одного поляризационного светоделителя PBS имеются два светоделителя $\mathrm{PBS}_{0}$ и $\mathrm{PBS}_{3}$ и вместо одного детектора $D_{0}$ - два детектора $D_{-0}$ и $D_{+0}$. Детектор

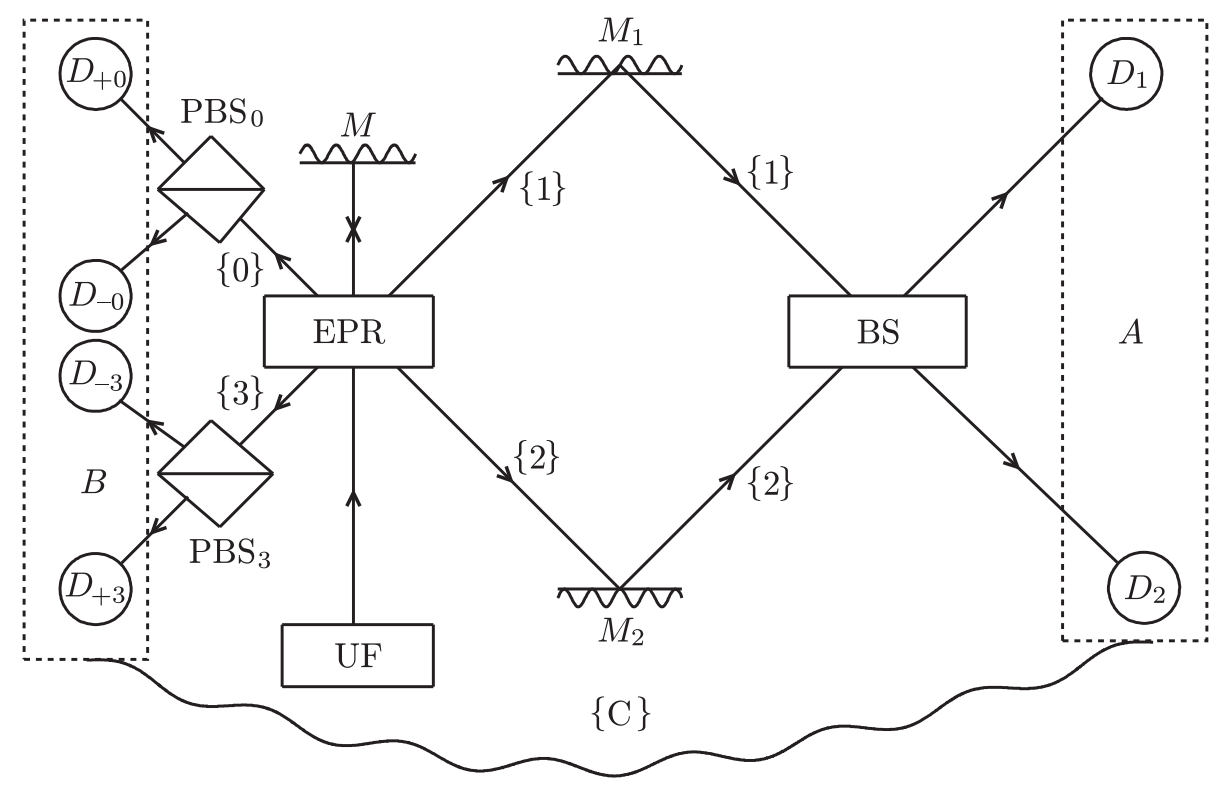

Рис. 5. Принципиальная схема эксперимента по телепортации запутанности.

$D_{+0}$ срабатывает, когда в поляризационном базисе светоделителя $\mathrm{PBS}_{0}$ фотон $\{0\}$ имеет горизонтальную поляризацию. Детектор $D_{-0}$ срабатывает, когда фотон $\{0\}$ имеет вертикальную поляризацию. Аналогичным образом работают детекторы $D_{+3}$ и $D_{-3}$, но их реакция, конечно, привязана к поляризационному базису светоделителя $\mathrm{PBS}_{3}$. 
В экспериментах изучались варианты совпадений $D_{1}-D_{2}-D_{ \pm 0}-D_{ \pm 3}$ при различных ориентациях поляризационных светоделителей по отношению к исходному поляризационному базису. Авторы экспериментов считали, что в случае, когда одновременно срабатывают детекторы $D_{1}$ и $D_{2}$, фотоны $\{1\}$ и $\{2\}$ оказываются в запутанном двухчастичном состоянии $\left|\Psi^{(-)}\right\rangle$. Поэтому согласно проекционному принципу квантовым двухчастичным состоянием фотонов $\{0\}$ и $\{3\}$ будет $\left|\Psi^{(-)}\right\rangle_{03}$. Таким образом, созданное с помощью светоделителя $\mathrm{BS}$ и детекторов $D_{1}$ и $D_{2}$ запутанное состояние фотонов $\{1\}$ и $\{2\}$ без всякого физического воздействия передается фотонам $\{0\}$ и $\{3\}$, которые были совершенно независимыми до манипуляций с фотонами $\{1\}$ и $\{2\}$.

В качестве доказательства того, что фотоны $\{0\}$ и $\{3\}$ действительно оказываются в квантовом состоянии $\left|\Psi^{(-)}\right\rangle_{03}$, рассматриваются следующие факты. При одинаковой ориентации поляризационных светоделителей $\mathrm{PBS}_{0}$ и $\mathrm{PBS}_{3}$ достигают максимумов четверные совпадения $D_{1}-D_{2}-D_{+0}-D_{-3}$ и $D_{1}-D_{2}-D_{-0}-D_{+3}$, а совпадения $D_{1}-D_{2}-D_{+0}-D_{+3}$ и $D_{1}-D_{2}-D_{-0}-D_{-3}$ достигают минимумов. Наоборот, когда светоделители $\mathrm{PBS}_{0}$ и $\mathrm{PBS}_{3}$ ортогональны друг другу, достигают максимумов совпадения $D_{1}-D_{2}-D_{+0}-D_{+3}$ и $D_{1}-D_{2}-D_{-0}-D_{-3}$, а минимумов - совпадения $D_{1}-D_{2}-D_{+0}-D_{-3}$ и $D_{1}-D_{2}-D_{-0}-D_{+3}$. При одновременном повороте поляризационных светоделителей на одинаковый угол максимумы остаются максимумами, а минимумы - минимумами. Этот факт интерпретировался как свидетельство сферической симметрии двухчастичного квантового состояния фотонов $\{0\}$ и $\{3\}$, что характерно для состояния $\left|\Psi^{(-)}\right\rangle_{03}$

Однако реальный эксперимент не показал полной сферической симметрии. Дело в том, что при описанном повороте осей поляризационных светоделителей глуби́ны максимумов и минимумов изменяются. Они достигают наибольших значений, когда оси поляризационных светоделителей $\mathrm{PBS}_{0}$ и $\mathrm{PBS}_{3}$ повернуты относительно исходного поляризационного базиса либо на $0^{\circ}$, либо на $90^{\circ}$. Наименьшие значения достигаются при $45^{\circ}$.

В рамках предлагаемого в данной статье подхода результаты подобных экспериментов можно интерпретировать способом, аналогичным описанному в разделе 5 . Простой светоделитель $\mathrm{BS}$ и детекторы $D_{1}$ и $D_{2}$ отбирают из множества различных пар фотонов $\{1\}$ и $\{2\}$ те пары, в которых фотоны в исходном поляризационном базисе имеют взаимно ортогональную поляризацию. Кроме того, среднее значение полного квазиспина у этих пар равно нулю. Так как элементарное состояние фотона $\{0\}$ является (негативной) копией элементарного состояния фотона $\{1\}$, а элементарное состояние фотона $\{2\}-$ (негативной) копией элементарного состояния фотона $\{3\}$, то одновременно происходит отбор пар фотонов $\{0\}$ и $\{3\}$, которые обладают теми же свойствами. Поэтому в случае, когда поляризационные светоделители $\mathrm{PBS}_{0}$ и $\mathrm{PBS}_{3}$ ориентированы по отношению к исходному базису под углом в $0^{\circ}$ или $90^{\circ}$, поляризации фотонов $\{0\}$ и $\{3\}$ в поляризационных базисах, связанных со светоделителями $\mathrm{PBS}_{0}$ и $\mathrm{PBS}_{3}$, должны быть ортогональными друг другу. В соответствии с этим должны наблюдаться те варианты совпадений, которые наблю- 
дались в эксперименте. Когда поляризационные делители $\mathrm{PBS}_{0}$ и $\mathrm{PBS}_{3}$ ориентированы под углом в $45^{\circ}$ относительно исходного базиса, можно провести рассуждения, которые проводились в разделе 5 . В результате мы придем к выводу, что при бесконечном числе испытаний в среднем поляризации фотонов $\{0\}$ и $\{3\}$, измеренные светоделителями $\mathrm{PBS}_{0}$ и $\mathrm{PBS}_{3}$, будут ортогональными. При небольшом количестве испытаний могут наблюдаться значительные отклонения от этого правила.

Таким образом, в описанном эксперименте в действительности происходит не столько телепортация запутанности, сколько отбор тех событий, в которых желаемая корреляция приближенно имеет место.

\section{7. ЗАКЛЮЧЕНИЕ}

Широко распространено мнение, что телепортация - это сугубо квантовый способ передачи информации, и этот способ может оказаться очень эффективным. Однако из приведенного в данной статье рассмотрения следует, что тесной связи телепортации с квантовостью может и не быть. Нетрудно привести классический аналог квантовой телепортации, давно применяемый на практике. Перед отплытием корабля его капитан получает от начальства запечатанный конверт, в котором содержатся несколько пронумерованных вариантов инструкции дальнейших действий. Когда корабль уходит далеко в море, капитан по радио получает приказ вскрыть конверт и выполнять инструкцию номер такой-то. В квантовой телепортации роль конверта выполняет частица из ЭПР-пары, а роль радио - классический канал связи.

В традиционный способ действия телепортация вносит некоторые изменения. Вместо одного конверта с инструкцией некоторый автомат изготавливает два идентичных конверта с инструкциями. В этих инструкциях варианты действий нумеруются одинаково, но случайным образом. Один конверт посылается капитану на корабле, другой - его начальству на берегу. До вскрытия конвертов никто не знает, какой номер имеет каждый из вариантов. Далее начальник вскрывает свой конверт, выбирает необходимый вариант и сообщает его номер капитану. По сравнению с традиционным такой способ действия имеет более высокую степень секретности. Однако за это преимущество приходится платить высокую цену. При традиционном способе действия конверт можно было вручить капитану до отплытия в море. При квантовой телепортации "конверт" посылается, когда капитан находится в море. Технически это может оказаться очень сложной задачей. Поэтому не следует особенно обольщаться возможностями квантовой телепортации.

\section{Список литературы}

[1] Д. Боумейстер, А. Экерт, А. Цайлингер (ред.), Физика квантовой информаиии, Постмаркет, М., 2002.

[2] Д. А. Славнов, ЭЧАЯ, 38:2 (2007), 295-359.

[3] Д. А. Славнов, ТМФ, 149:3 (2006), 457-472.

[4] Ж. Диксмье, $C^{*}$-алгебры и их представления, Наука, М., 1974.

[5] S. Kochen, E. P. Specker, J. Math. Mech., 17 (1967), 59-87.

4 Теоретическая и математическая физика, т. 157, № 1, 2008 г. 
[6] D. M. Greenberger, M. A. Horne, A. Shimony, A. Zeilinger, Amer. J. Phys., 58:12 (1990), 1131-1143.

[7] А.Н. Колмогоров, Основнъе понятия теории вероятностей, Наука, М., 1974.

[8] Ж. Неве, Математические основы теории вероятностей, Мир, М., 1969.

[9] J. S. Bell, Physics, 1:3 (1964), 195-200.

[10] J. S. Bell, "On the Einstein-Podolsky-Rosen Paradox", Speakable and Unspeakable in Quantum Mechanics: Collected Paper on Quantum Philosophy, Cambridge University Press, Cambridge, 1993, 139.

[11] J. F. Clauser, M. A. Horne, A. Shimony et al., Phys. Rev. Lett., 23:15 (1969), 880-884.

[12] Ж. Эмх, Алгебраические методы в статистической механике и квантовой теории поля, Мир, М., 1976.

[13] А. Эйнштейн, Б. Подольский, Н. Розен, УФН, 16:4 (1936), 440-446.

[14] Д. Бом, Квантовая теория, Наука, М., 1965.

[15] Д. Боумейстер, Х. Вайнфуртер, А. Цайлингер, "Протокол квантовой телепортации", Физика квантовой информации, ред. Д. Боумейстер, А. Экерт, А. Цайлингер, Постмаркет, М., 2002, 76-78.

[16] Д. Боумейстер, Дж.-В. Пэн, Х. Вайнфуртер, А. Цайлингер, “Эксперименты по квантовой телепортации кубитов", Физика квантовой информаиии, ред. Д. Боумейстер, А. Экерт, А. Цайлингер, Постмаркет, М., 2002, 95-103.

[17] T. Jennewein, G. Weihs, J-W. Pan et al., Phys. Rev. Lett., 88:1 (2001), 017903.

[18] Д. Боумейстер, Дж.-В. Пэн, Х. Вайнфуртер, А. Цайлингер, “Обмен перепутыванием: телепортация перепутывания", Физика квантовой информации, ред. Д. Боумейстер, А. Экерт, А. Цайлингер, Постмаркет, М., 2002, 116-119. 